\title{
The Clinical Analysis of Vaginal Delivery After Cesarean Section, and Favorable factors for Vaginal Delivery
}

\author{
Yanhua Liu, Guangpu Liu, Zheng Wang, Huixin Zhang \\ Department of Obstetrics and Gynecology, the Fourth Hospital of Hebei Medical University, Shijiazhuang, People's Republic of China \\ Email address: \\ 748926178@qq.com (Yanhua Liu), zhx6519@163.com (Huixin Zhang)

\section{To cite this article:} \\ Yanhua Liu, Guangpu Liu, Zheng Wang, Huixin Zhang. The Clinical Analysis of Vaginal Delivery After Cesarean Section, and Favorable \\ factors for Vaginal Delivery. Journal of Gynecology and Obstetrics. Vol. 6, No. 5, 2018, pp. 113-119. doi: 10.11648/j.jgo.20180605.12
}

Received: August 9, 2018; Accepted: September 9, 2018; Published: September 29, 2018

\begin{abstract}
The different modes of delivery and outcomes of mother and child in pregnant women who had prior cesarean section, and analysed favorable factors of vaginal delivery. Summarized characteristics related to vaginal delivery after cesarean section, in order to guide the clinic, decrease the rate of cesarean section, and promote natural childbirth. We designed an prospective study of pregnant women had single prior cesarean section (segmental transverse uterine scar), singleton, cephalic presentation, and full-term pregnancy, the total number was 379. Observed the mode of delivery and analysed the beneficial factors of vaginal delivery. Baseline maternal data and perinatal outcomes were recorded for a descriptive, and multivariate analysis. A p value $<0.05$ was considered significant. In this study, 87 cases pregnant women had trial of labor, 64 cases were vaginal delivery finally, the success rate was $73.56 \%$ (64/87). The maternal age, BMI index in pre-pregnancy and pregnancy, and blood loss were all smaller in women who had vaginal delivery than re-cesarean section $(p<0.05)$. The gestational weeks, cervical maturity, and interval time from the prior cesarean section were larger in women had vaginal delivery $(\mathrm{p}<0.05$ ). Multivariate analysis showed maternal age, BMI index in pre-pregnancy, cervical maturity and psychology were related to vaginal delivery. VBAC is feasible and safe. Most women had previous cesarean section refuse to trial of labor, often to choose re-cesarean section. So control the first indication of cesarean section is rather important in China. Attention women's weight before pregnant and psychology in pregnancy are significant. A management system of vaginal birth after cesarean were established, which to guide pregnant women more better and strengthen the education. In the meantime, have a good relationship and the trust between doctors and patients, pay attention to humanistic care, create a safe and comfortable environment to delivery should not be ignored.
\end{abstract}

Keywords: Trial of Labor, Vaginal Delivery After Cesarean Section, Mode of Delivery, Re-cesarean Section, BMI Index, Psychology

\section{Introduction}

Cesarean section is a effective medical measure for treatment of high risk pregnancy and dystocia, and non medical indication of cesarean section is increase gradually recently.

In particular, cesarean section rate has increased rapidly in China and WHO global survey showed the rate was 46.5\% [1]. Because of different levels of medical and relationships between doctors and patients are tensely, pregnant women choose re-cesarean section mostly. If not control the rate of cesarean section, it would increase to $56.2 \%$ [2].

Non medical indication of cesarean section is not approve the adverse outcomes of maternal and child, on the contrary, it increase the length of hospital stay, financial burden, the rate of neonatal wet lung syndrome and scar pregnancy. It also increase placenta previa and placenta accreta when the next pregnancy. So, the non indication of cesarean section should be controlled, and to promote the vaginal delivery.

Our country begun the "two children policy" in January 2016, it companied the number of pregnant women who had prior cesarean section increased. For the safe and health of maternal and child, a natural childbirth also should be encouraged.

\section{Materials and Methods}

An prospective study was designed including pregnant women who had single PCS (segmental transverse uterine scar). They were recruited in the Fourth Hospital of Hebei 
Medical University between September 2016 and September 2017 , both months included.

\subsection{Selection Criteria}

Pregnant women over $37^{+0}$ weeks of gestation with single PCS (segmental transverse uterine scar) with singleton gestation in cephalic presentation.

The condition of trial of labor for pregnant women had prior cesarean section:

(1) without serious complications influence vaginal delivery,

(2) the pelvis of maternal is normal,

(3) the indication of previous cesarean section is non exist,

(4) ultrasound indicate the muscle layer of the scar is continuous,

(5) the pregnant women and families support to trial of labor, understand the risks and to sign the consent form.

\subsection{Exclusion Criteria}

Women had history of transmural uterine surgery (different from segmental transverse cesarean section), more than one times of cesarean section.

\subsection{Statistical Methods}

Study data were collected from delivery information that recorded by the research team. Maternal and neonatal data were recorded and collected for posterior statistical analysis. IBM Statistics Process Social Sciences 21.0 for Mac was used.

Baseline maternal data and perinatal outcomes were recorded for a descriptive, factor analysis were used logistic regression. A p value $<0.05$ was considered statistically significant.

\subsection{Ethics Approval}

This study has the approval of the local ethics committee.

\section{Results}

\subsection{Recruitment, Baseline Data and Delivery Outcome}

The total number of delivery in our hospital was 1089 cases from September 2016 to September 2017, among them who had prior cesarean section were 379 cases, and meet the criteria of trial of labor were 87 cases, the rate of TOLAC was $22.96 \%$ (87/379), 64 cases were vaginal delivery finally, and the success rate of VBAC was $73.56 \%(64 / 87), 23$ cases were converted to re-cesarean section. Therefore, 87 women were included for multivariate analysis (Figure 1).

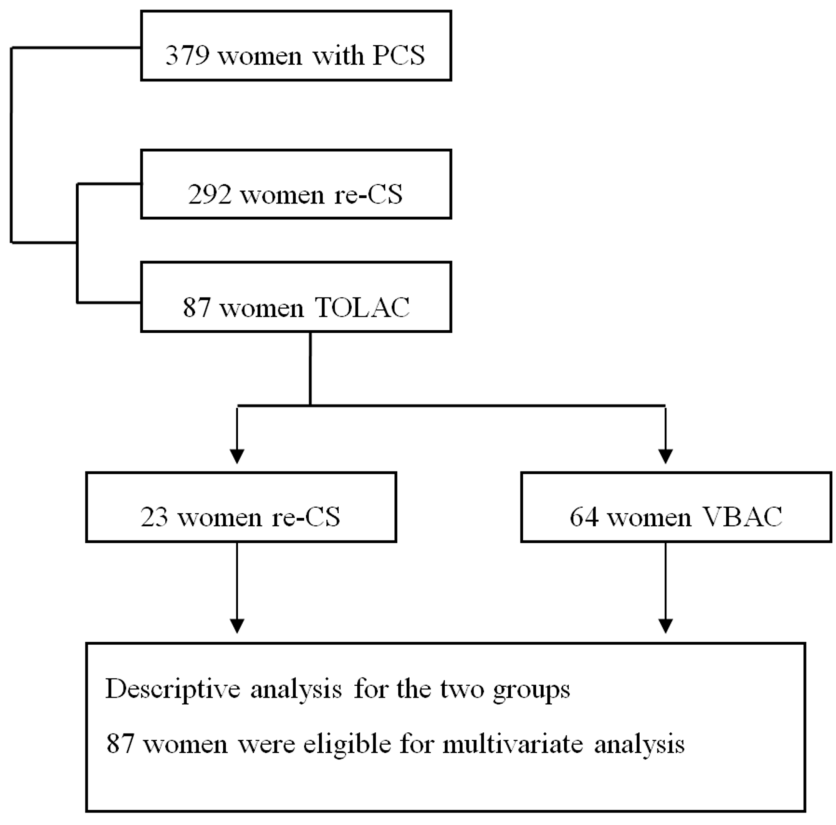

Figure 1. Recruitment and flow of women with TOLAC.

PCS: previous cesarean section

Re-CS: repeat cesarean section

TOLAC: trial of labor after cesarean section

VBAC: vaginal delivery after cesarean section

The main indications of re-cesarean section were social factors $(63 \%)$. The social factors were pregnant women and their families hadn't the desire of vaginal delivery, insist to had re-cesarean section.

\subsection{Compared the Basic Indexes Between the VBAC Group and Re-CS Group}

The maternal age was smaller in the VBAC group $[(28.38 \pm 2.86)$ vs. $(31.50 \pm 3.88)]$, the difference was significant. And gestational weeks in the VBAC group were more than re-CS group. While neonatal weight was no difference between the two groups. The maturity of cervical was higher in the VBAC group [(7.73 \pm 2.54$)$ vs. $(3.35 \pm 1.74)]$. (see Table 1.)

BMI index in Pre-pregnancy $[(21.28 \pm 2.18)$ vs. $(23.23 \pm 3.46)]$ and in pregnancy $[(26.77 \pm 2.82)$ vs. $(29.06 \pm 3.60)]$ were lower in the VBAC group, the difference were significant. However, the gain weight and BMI change values during pregnancy were no difference between the two groups. (see Table 1.)

Table 1. Clinical characteristics of pregnant women.

\begin{tabular}{lllll}
\hline Groups & Re-CS group Mean \pm SD & VBAC group Mean \pm SD & t & P value \\
\hline Maternal age & $31.50 \pm 3.88$ & $28.38 \pm 2.86$ & 6.28 & 0.010 \\
Gestational weeks & $38.74 \pm 1.40$ & $39.23 \pm 1.55$ & -2.12 & 0.020 \\
Neonatal weight & $3464.30 \pm 596.76$ & $3390.0 \pm 459.78$ & 0.62 & 0.426 \\
Pre pregnancy BMI & $23.23 \pm 3.46$ & $21.28 \pm 2.18$ & 8.47 & 0.000 \\
Gain weight & $15.12 \pm 4.78$ & $14.30 \pm 4.40$ & 1.17 & 0.276 \\
\hline
\end{tabular}




\begin{tabular}{lllll}
\hline Groups & Re-CS group Mean \pm SD & VBAC group Mean \pm SD & t & Pvalue \\
\hline Pregnancy BMI index & $29.06 \pm 3.60$ & $26.77 \pm 2.82$ & 5.57 & 0.018 \\
Change value of BMI & $5.82 \pm 1.82$ & $5.49 \pm 1.73$ & 0.88 & 0.346 \\
Bishop score & $3.35 \pm 1.74$ & $7.73 \pm 2.54$ & 17.14 & 0.000 \\
\hline
\end{tabular}

The blood loss was more in the VBAC group [450.0 (450.0, 600.0) $\mathrm{ml}$ vs. $265.0(220.0,367.5)$ ml], the difference was significant. The time interval between the two cesarean sections was longer in the re-CS group [6.0y (4.0, 7.0) vs. 4.0y (3.0, 6.0) ]. (Table 1, Table 2)

Table 2. Compared the blood loss and conditions of scare between two groups.

\begin{tabular}{llll}
\hline Groups & Blood loss $(\mathbf{m l})$ & $\begin{array}{l}\text { Interval time of operations } \\
\text { (year) }\end{array}$ & Scar thickness of lower uterine segment (mm) \\
\hline Re-CS group Median (interquartile range) & $450.0(450.0,600.0)$ & $6.0(4.0,7.0)$ & $49.0(38.0,50.0)$ \\
VBAC group Median (interquartile range) & $265.0(220.0,367.5)$ & $4.0(3.0,6.0)$ & $31.0(28.0,40.0)$ \\
Z & -9.582 & -3.125 & -1.853 \\
P value & 0.000 & 0.002 & 0.064 \\
\hline
\end{tabular}

Because of some datas about scar thickness of lower segment of uterus were missed, so we collected this datas were rather limited. The study revealed the scar thickness were no difference between two groups.

\subsection{Complications of Mother and Neonatal}

There was one case with rupture of uterine scar, and another one case with incompletely rupture of uterine scar in the re-CS group. In the VBAC group, there were some parturient women that bleeding continually from vaginal after delivery, and ultrasound revealed large blood accumulation in pelvic in that time. So we begun a exploratory laparotomy, discovered a scar dehiscence in the lower segment of uterus, and the scar dehiscence stretched to the right and down direction.

In the re-CS group, there had one neonatal's Apgar score was 7-10-10, the PH value of umbilical cord blood was 7.14 In the VBAC group, the number of low Apgar score were 2, the one newborn's Apgar score was 7-9-10, the PH value of umbilical cord blood was 7.02; the other one was forceps delivery and Apgar score was 5-7-9, the PH value of umbilical cord blood was 6.98 .

\subsection{Factors Conducive to Vaginal Delivery in Pregnant Women with Scarred Uterus}

This study showed maternal age, BMI index in pre-pregnant, cervical maturity and psychology were advantageous to VBAC. (Table 3,4)

Table 3. Assignment list of Logistic regression analysis.

\begin{tabular}{lll}
\hline factors & Variable name & Assignment description \\
\hline Maternal age & $\mathrm{X} 1$ & $<25=1,25-30=2,30-35=3,35-40=4,>40=5$ \\
Pre pregnancy BMI & $\mathrm{X} 2$ & $<18.5=1,18.5-22.9=2,23-24.9=3,25-29.9=4,>30=5$ \\
Gain weight $(\mathrm{kg})$ & $\mathrm{X} 3$ & $<12.5=1,12.5-17.5=2,>17.5=3$ \\
Bishop score & $\mathrm{X} 4$ & $<7=1, \geq 7=2$ \\
Psychology & $\mathrm{X} 5$ & Refuse to trial of labor=1, willingness to trial of labor=2 \\
Neonatal weight $(\mathrm{kg})$ & $\mathrm{X} 6$ & $<3.0=1,3.0-3.5=2,3.5-4.0=3,>4.0=5$ \\
The mode of delivery & $\mathrm{Y}$ & $\mathrm{CS}=1$, vaginal delivery=2 \\
\hline
\end{tabular}

Table 4. Multiple factor results of Logistic regression analysis.

\begin{tabular}{lllllll}
\hline & B & S.E, & Wals & Df & Sig. \\
\hline X1 & 2.483 & .781 & 10.118 & 1 & .001 \\
X2 & -4.456 & .997 & 19.968 & 1 & .000 \\
X4 & 3.948 & .535 & 54.507 & 1 & .977 & .012 \\
X5 & 2.682 & .967 & 7.691 & 1 & .000 & .006 \\
Constant & -9.655 & 1.861 & 26.907 & 1 & .000 \\
\hline
\end{tabular}

\section{Discussion}

At present, most women in childbearing age are the only child in their family, they were born from 1980s to the beginning of 1990 in China. They have a poor tolerance to pain, while part of them are over nutrition in pregnancy. Because some pregnant women lack exercise, their weight or fetal weight gain, and increase the difficult of vaginal delivery. In China, part of pregnant women choose cesarean section because of painful contractions. This makes the social factors of cesarean section in China still high. With the practice of "the policy of two children" in China since 2016, the number of pregnant women had history of cesarean section is increasing, so the relevant problems following, for example the mode of delivery. This study will explore the relevant problem, which are beneficial to clinical.

\subsection{Compared Characteristics Between the Two Groups}

In this study, the maternal age in the VBAC group was 
smaller than the re-CS group. Grobman discovered the maternal age had negative correlation with the success rate of VBAC (OR 0.96, 95\%CI 0.95-0.97) [3], and Malede had the same conclusion [4]. A large multicenter survey pointed out pregnant women whose age were less than 40 , the success rate of trial of labor was higher than the women whose age were more than 40 [5]. There has a study showed the risk of failed trial of labor wound increased by 1.22 times when maternal age increased 5 years each time [6].

This study displayed gestational weeks in the VBAC group were larger, and all the pregnant women were spontaneous labor, while most women request cesarean section before spontaneous labor in the re-CS group, so the gestational weeks were difference in the two groups. Mehmet also discovered gestational weeks were slightly larger in the VBAC group [7]. A retrospective study found no matter was spontaneous labor, or induction of labor, compared with women whose gestational weeks were less 40 , the success rate of VBAC of women whose gestational weeks more than 40 was lower [8]. In addition, Coassolo bringed 11000 pregnant women had trial of labor in the study, when controlled confounding factors, found the failed rate of VABC was higher for gestational weeks beyond 40 (31.3\% vs. 22.2\%; OR 1.36; 95\% CI, 1.24-1.50) [9]. In our study, more than 40 weeks accounted for $26.9 \%(14 / 52)$. Even though the rate of VBAC was higher when gestational weeks were more than 40 , but still encourage more pregnant women to have a try.

This paper displayed neonatal weight was no difference in the two groups, but smaller neonatal weight was beneficial to vaginal delivery. There had one study showed pregnant women had one previous cesarean section, with the increase of fetal weight, the success rate of VBAC showed a downward trend [10]. When fetal weight was more than $4000 \mathrm{~g}$, maybe the success rate of VBAC was less than $50 \%$, so the relationship of them was inverse ratio [11]. Lorie pointed out a lower birth weight had fewer failed VBAC [12]. However, it didn't show fetal weight had difference in the two groups. Therefore, women can trial of labor under the strict monitor.

In the VBAC group, BMI in pre-pregnant or in pregnant was less than the re-CS group, because the number of sample was limited, the maternal gain weight and the change value of BMI were no difference in the two groups. Hence, it can reflected the importance of maternal weight in pre-pregnant, maintained weight in the normal range were benefit to mother and neonatal.

According to one research, obesity reduced the success rate of VBAC, and controlled confounding factors, if BMI value was greater or equal to 30 in pregnancy, and BMI value was bigger in pre-pregnancy, then the success rate of VBAC decreased [13]. Regan found gain weight less than 30 pounds in pregnancy was benefit to VBAC [14]. And Callegari kept an account of pregnant women's BMI value in pre-pregnancy and BMI change value in pregnancy in Washington Regional Hospital during 1992-2009, the results showed compared with normal BMI in pre-pregnancy, and weight kept constant (BMI change value $<1$ ) in pregnancy, the success rate of VBAC would decrease $8 \%(\mathrm{RR}, 0.92 ; 95 \% \mathrm{CI}, 0.87-0.98)$ when gain weight increase moderately $(2>$ BMI change value $\geq 1)$; the rate would decrease $12 \%$ (RR, $0.88 ; 95 \% \mathrm{CI}, 0.83-0.93)$ when gain weight increase serious (BMI change value $\geq 2$ ); while lose weight in pregnancy was advantageous to VBAC for overweight or obesity [15]. Similar research revealed the success rate of trial of labor was more higher for pregnant women had previous cesarean section when BMI value was smaller [3].

In the VBAC group, Bishop score was more higher. The study of Mehmet showed disappearance of cervical canal (95\%CI: $1.06-1.14)$ and dilatation of uterine orifice $(95 \% \mathrm{CI}$ : 1.62-2.59) were important factors for VBAC [7]. Durnwald thought when dilatation of uterine orifice was more than $1 \mathrm{~cm}$, disappearance of cervical canal was more than $50 \%$, and the head of the fetus was located below the spine $-1 \mathrm{~cm}$, it was more easily to vaginal delivery [16]. One study showed dilatation of uterine orifice was bigger than $3 \mathrm{~cm}$, it was the most important factor to vaginal delivery [4]. While an another study revealed when dilatation of uterine orifice was bigger than $4 \mathrm{~cm}$, the success rate of VBAC was more higher, and meantime found compared with dilatation of uterine orifice was less than $100 \%$, the possibility success rate of VBAC wound increased 5 times for women whose cervical canal was complete disappeared [5]. While Kwon thought pregnant women had none indication of previous cesarean section, and in this delivery, the dilatation of uterine orifice was greater than or equal to $8 \mathrm{~cm}$ was an independent factor to predict the success of VBAC, and the rate was 93.1\% [17].

The amount of bleeding in cesarean section was significantly higher than in natural childbirth, because vaginal delivery could decreased the damage of tissue and avoid blood of intra-operate.

Interval time of prior operate in the re-CS group was longer $[6.0 \mathrm{y}(4.0,7.0)$ vs. $4.0 \mathrm{y}(3.0,6.0)]$. As the time of formation of scar goes on, the degree of scar degeneration increased, then tissue's elasticity decreased and brittleness increased. Therefore, Malede thought inter delivery interval was meaningless [4]. Due to a part of women hadn't measure the thickness of scar, so this data was limited, the total number were 61 cases, included 50 cases in the re-CS group and 11 cases in the VBAC group. This study hadn't showed the thickness of scar had difference between two groups. Yet, measure scare thickness was influenced by many factors, included technology, methods of measurement and filled degree of bladder, thus the accuracy was not high. The study of Nilanchali revealed measure scar at late second trimester and third trimester were important, it associated with the mode of delivery, and the thinner scar had more possibility had fetal bradycardia and meconium staining of liquor [18]. Meanwhile our study and the relevant literatures hadn't agree with significance of lower uterine segment thickness [19]. Especially, we have excluded the taboo of trial of labor and need to pay attention to the continuity of the muscular layer. If the continuity was poor, then the risk of uterine rupture is high, the women shouldn't to TOLAC. 


\subsection{The Favorable Factors to Vaginal Delivery after Cesarean Section}

We discovered maternal age, cervical maturity, BMI before pregnant and psychology were major factors influenced vaginal delivery. With the maternal age increased, the odds of complications were increased, the body functions were slightly reduced, so most of them reluctant to trial of labor. Mendoza also identified the maternal age was interrelated with VBAC [20, 21]. One study found once maternal age was more than 35, then the success of VBAC was decrease [21]. While Malede thought maternal age was not related with VBAC [4]. A study revealed cervical opening, effacement, gravidity, parity, and prior vaginal delivery were important factors for successful vaginal birth after cesarean section [7]. Another study showed maternal pre-pregnancy BMI, non-recurring indications of previous cesarean section, good Bishop's score at the time of admission, spontaneous onset of labor, and neonatal birth weight were associated with more higher probability of VBAC [22].

With enhance awareness of pregnancy examination regularly in China, gain weight of women control well mostly. This study found that normal BMI value in pre-pregnancy was advantages to VBAC, possibly due to limited data and no effect of weight gain on vaginal delivery. Vinturache discovered BMI value in pre-pregnancy was not determinants of modes of delivery for women had non history of cesarean section [23]. So combined with our study, the normal BMI value in pre-pregnancy was benefited to natural labor.

In this study, more than $50 \%$ women chosen re-CS, part of them were satisfied with the condition of trial of labor and the possibility of vaginal delivery was high, but still consist to choose re-CS, So psychology of pregnant women was rather important. Then doctor need to inform contraindication of VBAC and relevant risks, identified whether women could undertake it. In the meantime, give women more confidence in natural birth and relieve the fears. In the process of delivery, the midwife nursing not only strengthen confidence, but also could shorten the duration of labor and decrease the blood loss [24]. Goldman thought doctor's attitude was the major factor influenced the VBAC [25]. We consider pregnant women obtain the trust, support and understand are more benefits for VBAC.

Especially, women need receive the relevant knowledge about vaginal delivery after cesarean section in the pregnancy period. Let them know the advantages and risks, so they can manage themselves in pregnancy more better. Now, more pregnant women gained information and experiences what they concerned from social media, it was convenient and low-cost [26]. Shorten [27] thought construct website about vaginal delivery after cesarean section actively at the premise of protect personal privacy and the security, help pregnant women choose reasonable mode of delivery preliminarily. The website should include detailed personal information, various indexes during pregnancy and some tests can understand the maternal preference and psychological activity. The prominent position of the website should introduce science knowledge about VBAC, provide communication platform and share experiences. And Konheim found most pregnant women obtained knowledge and experience from online discussion module, more than a third of discussion area had somebody described the process of delivery, while these contents rarely appeared in the normal discussion area [28]. Construct discussion area of website positively, let more women participate in it, obtain more feedbacks from it. So understand the questions what the pregnant women ask, to meet demand of them. In other hand, Susan found most women hadn't enough counseled about delivery and it was affected by many factors [29]. All things considered, give them more supports and encouragements, strengthen the confidence of vaginal delivery, increase care and decrease the fear [30].

There had one study showed the clinician's guidelines was influenced the VBAC, include aspects mentioned above, for example counseling, give women more confidence, support and trust, let women consider VBAC as the first choice and understand the meaning of birth naturally [31]. Pregnant women anticipated VBAC played a key role in vaginal delivery [32].

\subsection{The Characteristic of VBAC in this Study}

In the VBAC group, the percentage of women whose prior cesarean section was done in tertiary hospital was $76.9 \%$ (40/52), then we can reveal the more grade of hospital, the more higher standard of operate and the more secure of uterine incision in China, thus, the more higher security of trial of labor after cesarean section.

We found regular uterine contraction and good maturity of cervix were the major factors to promote VBAC, and Landon, Macones had similar viewpoints with us $[33,34]$.

There were 9 cases pregnant women had prior trial of labor in the VBAC group. Scott revealed women had prior trial of labor, no matter it success or fail, all had advantages to this delivery [35].

\subsection{Attention the Sign of Uterine Rupture and How to Deal with it in the Process of TOLAC}

This study showed the successful rate of VBAC was $70.3 \%$. Once women had trial of labor after cesarean section, we need to consider the risk of uterine rupture. However, it was not easy to discover the sign of uterine rupture, it maybe showed tenderness of the scar or the abdomen, rebound pain, muscle tension and so on. Some fetal heart monitoring could presented abnormal changes, maybe accompanied by maternal heart rate increase, or abdominal distension, hemoglobin dropped or the ultrasound pointed out abnormal change.

The study of Holmgren discovered expulsion of the fetus within 18 minutes when happened suspect signs of uterine rupture, then most neonates' $\mathrm{PH}$ of umbilical artery blood were in the normal range and Apgar score in $5 \mathrm{~min}$ were more than 7. So, It's important to deal with it in time [36]. 


\section{Conclusions}

VBAC is feasible and safe, not increase the rate of postpartum hemorrhage and neonatal asphyxia. Pre-pregnancy BMI index, the maturity of cervical and psychological factors are relate with VBAC closely.

We should establish the management of VBAC, to guide pregnancy, strengthen the education of $\mathrm{VBAC}$, and have a good relationship between doctor and patient, increasing the trust between them, paying attention to humanistic care, creating a safe and comfortable environment to delivery. The strict monitoring system and the ability of emergency cesarean section are important to ensure the safety of mother and baby.

\section{Limitations}

Unfortunately, the sample size was limited to compared the difference between VBAC group and re-CS group. In this study, the number of women who had vaginal delivery after cesarean section was less. Besides, the thickness of uterine scare didn't measured routinely in this study, so compared this index was not reasonable between the two groups. On the other hand, the results in this paper were assisted in this hospital, and results of the process maybe different in other centers with a change in clinical approach.

\section{References}

[1] Lumbiganon P, Laopaiboon M, Gulmezoglu AM. Method of delivery and pregnancy outcomes in Asia: the WHO global survey on maternal and perinatal health 2007-2008. Lancet, 2010; 375 (9):490-499.

[2] Scott JR. Vaginal birth after cesarean: a common sense approach. Obstet Gynecol, 2011; 118 (pt 1):342-350.

[3] Grobman WA, Lai Y, Landon MB. Development of a nomogram for prediction of vaginal birth after cesarean delivery”. Obstet Gynecol; 2007, 109 (4):806-812.

[4] Malede Birara, Yirgu Gebrehiwot. Factors associated with success of vaginal birth after one caesarean section (VBAC) at three teaching hospitals in Addis Ababa, Ethiopia: a case control study. BMC Pregnancy and Childbirth, 2013; 13, 31.

[5] Flamm BL, Geiger AM. Vaginal birth after cesarean delivery: an admission scoring system. Obstet Gynecol, 1997;90 (6):907-910.

[6] Smith GC, Pell JP, Pasupathy D. Factors predisposing perinatal death related to uterine rupture during attempted vaginal birth after caesarean section. BMJ, 2004; 329, 375.

[7] Mehmet Baki Senturk, Yusuf Cakmak, Halit Atac. Factors associated with successful vaginal birth after cesarean section and outcomes in rural area of Anatolia. International Journal of Women's health, 2015; 7:693-697.

[8] Zelop CM, Shipp TD, Cohen A, Repke JT, Lieberman E. Trial of labor after 40 weeks' gestation in women with prior cesarean. Obstet Gynecol, 2001; 97, 391-393.

[9] Coassolo KM, Stamilio DM, Pare E. Safety and efficacy of vaginal birth after cesarean attempts at or beyond 40 weeks of gestation. Obstet Gynecol, 2005; 106:700.
[10] Elkousky MA, Samuel M, Stevens E. The effect of birth weight on vaginal birth after cesarean delivery success rates. Am J Obstet Gynecol, 2003; 188:824-830.

[11] Zelop CM, Shipp TD, Repke JT. Outcomes of trial of labor following previous cesarean delivery among women with fetuses weighing $>4000$ g. Am J Obstet Gynecol, 2001;185 (4):903-905.

[12] Lorie M. HARPER, David M. STAMILIO, Anthony O. ODIBO, Jeffrey F. PEIPERT. Vaginal birth after cesarean for cephalopelvic disproportion: effect of birth-weight difference on success. Obstet Gynecol, 2011; 117 (2 Pt 1):343-348.

[13] Durnwald CP, Ehrenberg HM, Mercer BM. The impact of maternal obesity and weight gain on vaginal birth after cesarean section success. Am J Obstet Gynecol, 2004;191 (3):954-957.

[14] Regan J, Keup C, Wolfe K. Vaginal birth after cesarean success in high-risk women: a population-based study. J Perinatol, $2015 ; 35$ (4):252-257.

[15] Callegari LS, Sterling LA, Zelek ST. Interpregnancy body mass index change and success of term vaginal birth after cesarean delivery. Am J Obstet Gynecol, 2014; 210 (4):330. e1-7.

[16] Durnwald C, Mercer B. Vaginal birth after Cesarean delivery: predicting success, risks of failure. JMatern Fetal Neonatal Med, 2004; 15 (6):388-93.

[17] Kwon JY, Jo YS, Lee GS. Cervical dilatation at the time of cesarean section may affect the success of a subsequent vaginal delivery. Maternal Fetal Neonatal Med, 2009; 22 (11):1057-1062.

[18] Nilanchali Singh, Reva Tripathi, Y. M. Mala, Rashmi Dixit. Scar thickness measurement by transvaginal sonography in late second trimester and third trimester in pregnant patients with previous cesarean section: does sequential change in scar thickness with gestational age correlate with mode of delivery? J Ultrasound, 2015; 18 (2):173-178.

[19] Sentilhes L, Vayssiere C, Beucher G. Delivery for women with a previous cesarean: guideline for clinical practice from the French College of Gynecologists and Obstetricians (CNGOF). Eur J Obstet Gynecol Reprond Biol, 2013;170:25-32.

[20] Srinivas SK, Stamilio DM, Sammel MD. Vaginal birth after caesarean delivery: does maternal age affect safety and success? Paediatr Perinat Epidemiol, 2007; 21 (2):114-120.

[21] Sepúlveda-Mendoza DL, Galván-Caudillo M, Soto-Fuenzalida GA. Factors associated with successful vaginal birth in women with a cesarean section history. Ginecol Obstet Mex, 2015; 83 (12): 743-749.

[22] Smriti Gupta, Shanti Jeeyaselan, Raka Guleria, Anjali Gupta. An observational study of various predictors of success of vaginal delivery following a previous cesarean section. J Obstet Gynaecol India, 2014; 64 (4):260-264.

[23] Vinturache A, Moledina N, McDonald S. Pre-pregnancy Body Mass Index (BMI) and delivery outcomes in a Canadian population. BMC Pregnancy Childbirth, 2014; 20 (14):422.

[24] Zhang T, Liu C. Comparison between continuing midwifery care and standard maternity care in vaginal birth after cesarean. Pak J Med Sci.2016; 32 (3):711-714. 
[25] Goldman G, Pineault R, Potvin L. Factors Influencing the practice of vaginal birth after cesarean section. Am J Public Health, 1993; 83 (8): 1104-1108.

[26] Amy M. Romano, Hilary Gerber, Desirre Andrews. Social media, power, and the future of VBAC. J Perinat Educ, 2001; $19(3): 43-52$.

[27] Shorten A, Fagerlin A, llluzzi J. Developing an Internet-Based Decision Aid for Women Choosing Between Vaginal Birth After Cesarean and Planned Repeat Cesarean. Midwifery Womens Health, 2015; 60 (4): 390-400.

[28] Konheim-Kalkstein YL, Whyte R. What are VBAC Women Seeking and Sharing? A Content Analysis of Online Discussion Boards. Birth, 2015; 42 (3):277-282.

[29] Susan Folsom, M Sean Esplin, Sean Edmunds, Torri D. Metz. Patient counseling and preference for elective repeat cesarean delivery. AJP Rep, 2016; 6 (2):e226-e231.

[30] Ingela Lundgren, Patricia Healy, Margaret Carroll, Cecily Begley, Andrea Matterne. Clinicians' views of factors of importance for improving the rate of VBAC (vaginal birth after caesarean section): a study from countries with low VBAC rates. BMC Pregnancy Childbirth, 2016; 16, 350 .

[31] Ingela Lundgren, Evelien van Limbeek, Katri
Vehvilainen-Julkunen. Clinicians' views of factors of importance for improving the rate of VBAC (vaginal birth after caesarean section): a qualitative study from countries with high VBAC rates. BMC Pregnancy Childbirth, 2015; 15, 196.

[32] Mairead Black, Vikki A Entwistle, Silaitya Bhattacharya, Kaitie Gillies. Vaginal birth after caesarean section: why is uptake so low? Insights from a meta-ethnographic synthesis of women's accounts of their birth choices. BMJ Open, 2016;6, 1 .

[33] Landon MB, Hauth JC, Leveno KJ. Maternal and perinatal outcomes associated with a trial of labor after prior cesarean delivery. N Engl J Med, 2004; 351 (25): 2581-2589.

[34] Macones GA, Peipert J, Nelson DB. Maternal complications with vaginal birth after cesarean delivery: a multicenter study. Am J Obstet Gynecol, 2005; 193 (5): 1656-1662.

[35] Scott JR. Intrapartum management of trial of labor after caesarean delivery: evidence and experience. Bjogan International Journal of Obstetrics and Gynaecology, 2014; 121 (2): $157-162$.

[36] Holmgren C, Scott JR, Porter TF. Uterine rupture with attempted vaginal birth after cesarean delivery: decision-to-delivery time and neonatal outcome. Obstet Gynecol, 2012; 119 (4): 725-731. 